\title{
Transmission d'une impulsion de tension dans une colonne d'eau
}

\author{
C. Favreau \\ Centre d'Etudes Nucléaires, Service des Transferts Thermiques, 85 X, 38041 Grenoble Cedex, France
}

(Reçu le 5 janvier 1984, révisé le 19 juillet, accepté le 31 juillet 1984)

\begin{abstract}
Résumé. - On décrit le dispositif expérimental qui permet d'étudier la transmission d'une impulsion de tension (ou impulsion de pression négative) appliquée directement à une colonne d'eau. On montre que si, pour les amplitudes faibles, le signal transmis est proportionnel au signal appliqué, il subit aux amplitudes plus élevées une déformation caractéristique; l'amplitude transmise continue cependant à croître avec l'amplitude appliquée, bien que plus lentement. On étudie cette déformation qui se traduit, en particulier, par une chute brutale de la tension au cours de sa croissance, et l'on montre que l'amplitude transmise dépend alors non seulement de l'amplitude appliquée, mais de sa vitesse de montée. Pour rendre compte de ce comportement, on propose un modèle basé sur la croissance vaporeuse des microbulles en suspension au sein du liquide.
\end{abstract}

\begin{abstract}
An apparatus, permitting the study of the transmission of a tension (i.e. negative pressure) pulse directly applied to a column of water, is described. It is experimentally shown that, at low amplitudes, the applied and transmitted signals are proportional, and that at higher amplitudes, a characteristic deformation of the transmitted signal involves a slower rate of increase of its amplitude. This deformation, primarily described as a sharp drop of the tension during its growth, is studied. It is demonstrated that the amplitude transmitted is related not only to the amplitude applied, but to its rate of increase. To explain this behaviour, a model based on the vaporous growth of microbubbles suspended in the bulk of the liquid is proposed.
\end{abstract}

\section{Nomenclature}

$a$ atténuation dans les barres

$b$ coefficient d'atténuation linéique dans la colonne d'eau

$B$ module d'élasticité volumique du liquide

$C$ vitesse de transmission

$D$ diamètre

$E$ module d'Young

$f$ facteur de contrainte adimensionnel

$F$ fréquence

$h$ épaisseur de paroi

I interface entre barre et liquide

$k$ facteur de transmission entre les capteurs

$K$ coefficient de transmission ou réflexion aux interfaces

$K$ coefficient de compressibilité

$L$ longueur

$m$ pente de montée de tension

$p$ tension instantanée

$P$ amplitude de $p$

$r$ rayon de particules en suspension

$R$ rayon de bulles

s signal instantané provenant d'un capteur

$S$ amplitude de $\mathrm{s}$

$T$ temps de montée de la tension
$Z$ impédance acoustique

$\alpha$ fraction de vide instantanée

$\beta$ exposant dans la loi de distribution de $R_{0}$

$\lambda$ longueur d'onde

$\rho$ masse volumique

$\tau$ délai de transmission

\section{Indices}

0 valeur initiale

1 relatif à la barre émettrice entre capteur et liquide

2 relatif à la barre réceptrice entre capteur et liquide

b relatif au matériau des barres

c relatif au matériau de la chambre

g gaz

i impulsion incidente

$\ell$ liquide

$M$ valeur maximum

$r$ réflexion à une interface

$t$ transmission à une interface

\section{Introduction.}

De nombreux problèmes de dynamique des fluides nécessitent la modélisation du comportement d'un liquide réel soumis à une tension (ou pression négative) transitoire; cette tension est généralement provoquée 
par la réflexion d'une onde de compression sur une surface libre; mentionnons à titre d'exemples les effets de surface des explosions sous-marines et les contraintes dans un réservoir lors d'une secousse sismique ou d'une explosion interne.

Depuis le milieu du siècle dernier, les études expérimentales du comportement des liquides sous tension se sont diversifiées; les méthodes d'application de la tension peuvent toutefois se répartir en trois catégories :

- Les méthodes statiques : la tension est appliquée progressivement, sur une échelle de temps de plusieurs secondes ou minutes. Les plus connues sont la méthode du tube de Berthelot, ainsi que la méthode centrifuge qui permit à Briggs [1] d'atteindre le maximum pour l'eau de - 277 bar.

- Les méthodes ultrasoniques : la tension est appliquée au cours des alternances négatives d'une onde acoustique alternative périodique. Le maximum pour l'eau, - 380 bar, a été atteint par Syrotyuk [2] à l'aide d'un champ ultrasonique focalisé de $500 \mathrm{kHz}$.

- Les méthodes impulsionnelles : la tension est appliquée pendant un bref intervalle de temps, de l'ordre de la milliseconde ou moins. La méthode du piston a conduit Brown [3] à la valeur maximum pour l'eau de - 46 bar, à l'aide d'une impulsion en arche de sinusoïde de $20 \mu$ s de temps de montée.

Notre étude expérimentale utilise elle aussi la méthode du piston; cependant, contrairement à la quasi-totalité des études antérieures qui obtiennent la tension par réflexion d'une impulsion de compression sur une surface libre, c'est directement une impulsion de tension que nous appliquons au liquide.

\section{Dispositif expérimental (Fig. 1).}

Le liquide est contenu dans une chambre cylindrique de $16 \mathrm{~mm}$ de diamètre, forée dans un bloc en plexiglas de section carrée de $60 \mathrm{~mm}$ de côté ; la colonne liquide est délimitée à ses extrémités par deux barres en alliage d'aluminium de même diamètre que la chambre; deux paires de joints toriques encastrés dans la paroi de la chambre assurent l'étanchéité au niveau de chaque barre. Les parois latérales extérieures du bloc de plexiglas sont en contact avec un fluide thermostatique permettant d'amener éventuellement le liquide à une température supérieure à l'ambiante.

Pour effectuer le remplissage de la chambre, l'extrémité inférieure de la barre supérieure est amenée au niveau de deux ouvertures situées à la partie supérieure de la chambre; le liquide est alors introduit par l'une de ces ouvertures, l'air s'échappant à mesure -par l'autre ; l'air résiduel est enfin chassé par ces mêmes ouvertures en remontant légèrement la barre inférieure dans la chambre. La barre inférieure, d'une longueur de $1 \mathrm{~m}$, sert de guide à une masselotte annulaire et comporte à sa base une butée. L'impact de la masselotte sur la butée engendre dans la barre une impulsion de tension qui est transmise (partiellement) au liquide.

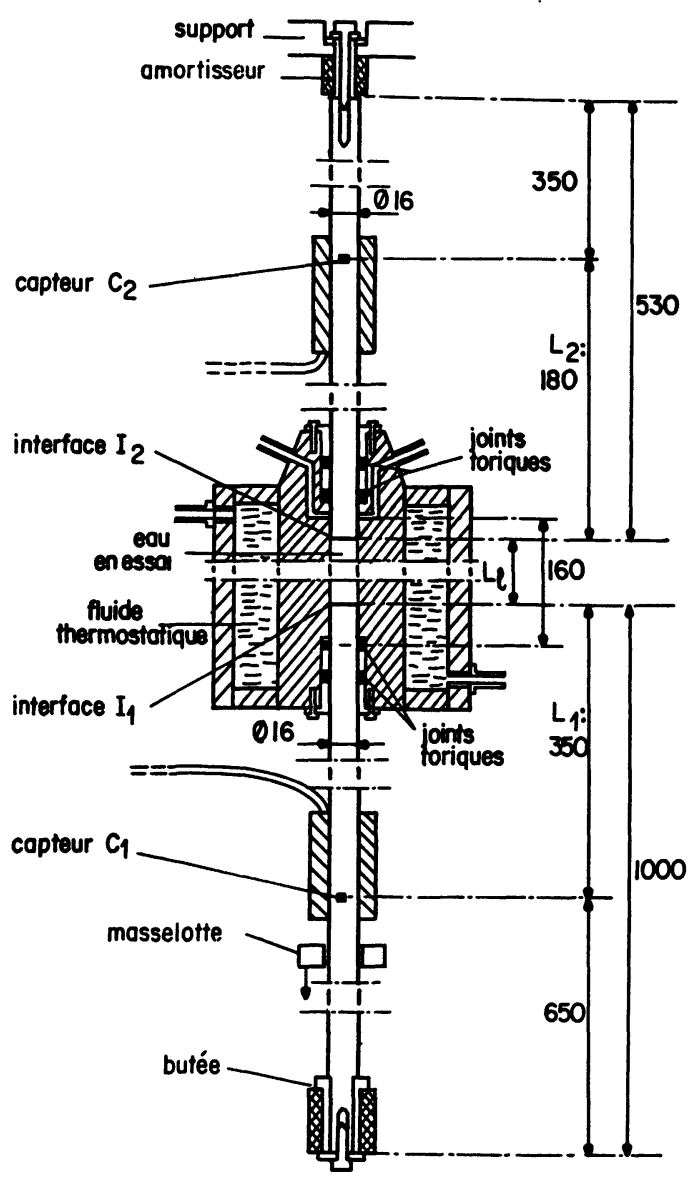

Fig. 1. - Dispositif d'essai. Dimensions en $\mathrm{mm}$.

[Test section. Dimensions in $\mathrm{mm}$.]

La forme de l'impulsion dépend de la nature et de la longueur de la masselotte; pour une masselotte donnée, l'amplitude de l'impulsion est fonction directe de sa hauteur de chute; la détection de l'impulsion dans la barre est assurée par un capteur de contrainte miniature $C_{1}$ collé à la distance $L_{1}=35 \mathrm{~cm}$ de la base de la colonne liquide.

La barre supérieure qui reçoit l'impulsion transmise par la colonne liquide a une longueur de $53 \mathrm{~cm}$; la détection de l'impulsion dans la barre est assurée par un capteur $C_{2}$ identique au précédent collé à la distance $L_{2}=18 \mathrm{~cm}$ du sommet de la colonne liquide; un amortisseur composite assure un bon isolement acoustique entre la barre et son support.

Chaque capteur est constitué de 4 jauges de contrainte miniatures (VISHAY EA 13-031 DE-120) en alliage de constantan, de longueur utile $0,79 \mathrm{~mm}$, deux d'entre elles étant collées longitudinalement sur la barre, les deux autres transversalement; elles sont interconnectées électriquement selon un montage en pont classique à 4 branches actives; alimenté par une source de tension continue stabilisée de $5 \mathrm{~V}$, ce pont a une sensibilité de $10 \mu \mathrm{V} /$ bar.

Les signaux électriques délivrés par les capteurs sont conditionnés par des amplificateurs différentiels 
(PRESTON 8300 XWB) qui assurent l'isolement électrique entre entrée et sortie, et qui ont un gain de 1000 et une bande passante de $100 \mathrm{kHz}$; un filtre passe-bas élémentaire de $50 \mathrm{kHz}$ de bande passante a été adjoint à leurs sorties afin de réduire leur dépassement transitoire (overshoot) et diminuer leur bruit de démodulation.

L'enregistrement des signaux des capteurs est effectué par des enregistreurs de transitoires à mémoire numérique (DATALAB DL 905), dont la bande passante est de $3 \mathrm{MHz}$ et les résolutions en amplitude et temporelle de, respectivement, $0,4 \%$ de la pleine échelle et $0,1 \%$ de la durée d'acquisition, ajustable de $0,2 \mu \mathrm{s}$ à $10 \mathrm{~s}$.

Les signaux sont ensuite retranscrits à faible vitesse sur un enregistreur graphique classique (SEFRAM P.E.D.).

\section{Eléments de base d'interprétation des signaux.}

3.1 Signal De L'impulsion initiale (Fig. 2). Lorsque, à la suite d'un impact, l'impulsion de tension initiale qui se propage dans la barre inférieure atteint le capteur $C_{1}$, celui-ci délivre un signal qui présente un front de montée, quasi linéaire sur la plus grande partie de sa croissance, suivi d'une décroissance plus lente, de forme grossièrement exponentielle, à laquelle peuvent se superposer des oscillations; celles-ci, qui apparaissent surtout lorsque la masselotte a une longueur supérieure à $1 \mathrm{~cm}$, peuvent être attribuées à des réflexions multiples au niveau de la butée, ainsi qu'à la réponse intrinsèque de la barre, comme l'a montré Davies [4].

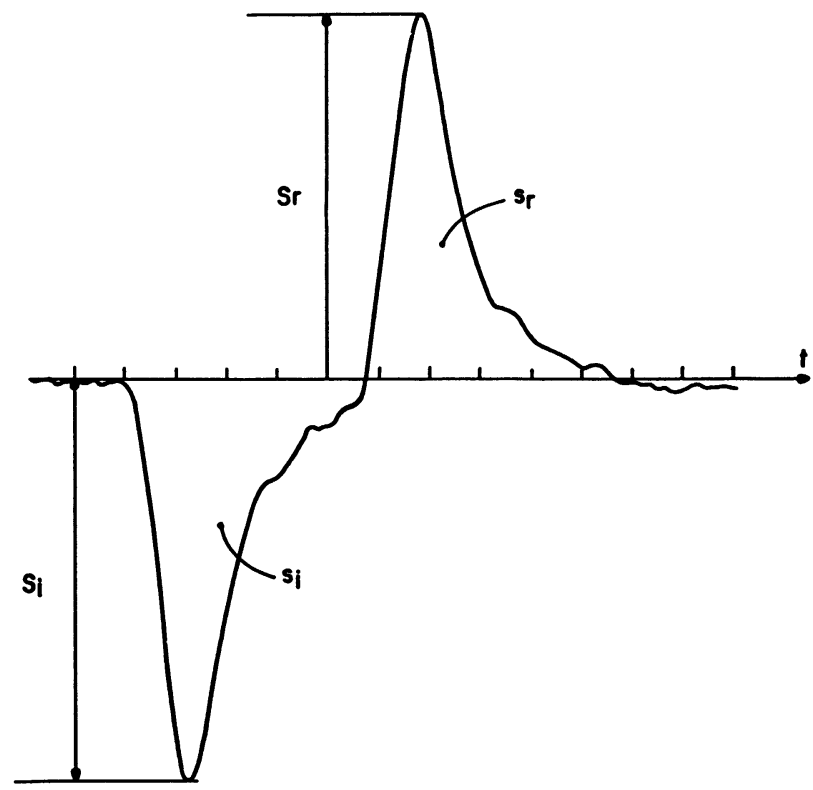

Fig. 2. - Signal typique sur le capteur de la barre émettrice obtenu avec une masselotte de $1 \mathrm{~cm}$ de long. Echelle de temps : $30 \mu \mathrm{s} /$ division.

[Typical signal from the transducer of the emitting rod, obtained with a weight $1 \mathrm{~cm}$ long. Time scale : $30 \mu \mathrm{s} /$ division].
Pour caractériser le signal $\mathrm{s}$, nous utiliserons deux paramètres : l'amplitude $S$ et le temps de montée $T$. La gamme d'amplitude couverte au cours des différents essais est de 0 à -250 bar environ au niveau du capteur $\mathrm{C}_{1}$. La valeur la plus faible de $T$, de l'ordre de $20 \mu$ s, est obtenue par l'utilisation de la masselotte de moindre longueur, soit $1 \mathrm{~cm}$. Notons que, pour cette forme de signal, la densité spectrale devient pratiquement négligeable au-delà d'une fréquence $F_{\mathrm{M}}$ telle que :

$$
F_{\mathrm{M}}=\frac{1}{T} \sim 50 \mathrm{kHz}
$$

3.2 TRANSMISSION DANS LES BARRES. - La théorie élémentaire de la prọpagation d'une onde longitudinale dans une barre cylindrique élastique de module d'Young $E_{\mathrm{b}}$ et de masse volumique $\rho_{\mathrm{b}}$ conduit à une vitesse de propagation $C_{\mathrm{b}}=\left(E_{\mathrm{b}} / \rho_{\mathrm{b}}\right)^{1 / 2}$ indépendante de la fréquence; ceci n'est valable en toute rigueur qu'asymptotiquement lorsque le rapport $\left(D / \lambda_{\mathrm{b}}\right) \mathrm{du}$ diamètre de la barre à la longueur d'onde dans celle-ci est nul. Dans notre cas, la valeur de ce rapport reste nettement inférieure à l'unité; sa valeur maximale est, en effet, de l'ordre de 0,16 .

Dans ces conditions, on peut montrer, à partir de l'étude de Davies, que la dispersion dans les barres et la distorsion qui en découle restent faibles. Pour le vérifier, on peut comparer le signal réfléchi $s_{r}$ dans la barre inférieure au signal incident $s_{i}$ lorsque la chambre ne contient pas de liquide; dans ce cas en effet (cf. § 3.4), la réflexion sur l'extrémité supérieure de la barre est totale. On constate effectivement que, lorsque le signal est assez bref pour que la fin du signal incident n'empiète pas sensiblement sur le signal réfléchi (Fig. 2). il ne présente pas de distorsion appréciable. La mesure des amplitudes, $S_{\mathrm{i}}$ et $S_{\mathrm{r}}$, de $\mathrm{s}_{\mathrm{i}}$ et $\mathrm{s}_{\mathrm{r}}$ fait apparaître une légère atténuation $a$ :

$$
a=\left|S_{\mathrm{r}} / S_{\mathrm{i}}\right| \sim 0,92
$$

on en déduit, sur les parcours $L_{1}$ et $L_{2}$ les atténuations $a_{1} \sim 0,96$ et $a_{2} \sim 0,98$.

La mesure de l'intervalle de temps $\tau_{1}$ entre les maxima de $s_{i}$ et $s_{r}$ de $138,0 \mu \mathrm{s}$, permet de calculer la vitesse de propagation $C_{\mathrm{b}}$ dans la barre :

$$
C_{\mathrm{b}}=2 L_{1} / \tau_{1}=\frac{2 \times 0,35 \mathrm{~m}}{138 \times 10^{-6} \mathrm{~s}}=5072 \mathrm{~m} / \mathrm{s}
$$

valeur proche de la vitesse théorique à basse fréquence dans une barre d'aluminium $(E=70 \mathrm{GPa}$, $\rho=2700 \mathrm{~kg} / \mathrm{m}^{3}$ ) pour laquelle on obtient $5091 \mathrm{~m} / \mathrm{s}$.

3.3 Transmission dans la colonNe D'eau. - La théorie élémentaire de la propagation d'une onde longitudinale dans une colonne liquide de module d'élasticité volumique $B$ et de masse volumique $\rho_{\rho}$, contenue dans une conduite élastique de module d'Young $E_{\mathrm{c}}$, conduit à une vitesse de propagation $C_{\ell}$ 
indépendante de la fréquence, mais plus faible que la vitesse d'onde plane $C_{\ell_{0}}=\left(B / \rho_{\ell}\right)^{1 / 2}$ :

$$
C_{\ell}=\frac{C_{\ell_{0}}}{\left[1+f \cdot\left(B / E_{\mathrm{c}}\right)\right]^{1 / 2}}
$$

où $f$ est un facteur de contrainte adimensionnel qui dépend des caractéristiques géométriques et physiques de la conduite. Dans le cas d'une conduite cylindrique de diamètres externe et interne $D_{\mathrm{e}}$ et $D_{\mathrm{i}}$ respectivement, Paynter [5] donne l'expression de $f$ suivante :

$$
f=2 \frac{D_{\mathrm{e}}^{2}+D_{\mathrm{i}}^{2}}{D_{\mathrm{e}}^{2}-D_{\mathrm{i}}^{2}}
$$

que l'on peut exprimer en fonction du diamètre moyen $D=\left(D_{\mathrm{e}}+D_{\mathrm{i}}\right) / 2$ et de l'épaisseur de paroi $h$ de la conduite :

$$
f=h / D+D / h \text {. }
$$

On vérifie ainsi facilement que, lorsque $h / D$ tend vers zéro, $C_{\ell}$ tend vers l'expression classique de la vitesse du coup de bélier, dite de Joukowski :

$$
C_{\mathrm{J}}=\frac{C_{\ell_{0}}}{\left[1+\frac{D}{h}\left(B / E_{\mathrm{c}}\right)\right]^{1 / 2}} .
$$

Les études théorique de Rubinov et Keller [6] et expérimentale de Barez et al. [7] montrent qu'une dispersion apparaît lorsque l'on introduit le comportement dynamique du tube (et particulièrement l'inertie radiale) ainsi que ses propriétés visco-élastiques. De même que dans le cas de la propagation dans les barres, la dispersion se traduit par une déformation progressive de l'impulsion au cours de son déplacement dans la colonne liquide, caractérisée par une diminution d'amplitude et un certain étalement.

Ce comportement a été mis en évidence à l'aide d'impulsions de compression, afin de s'affranchir des effets éventuels de cavitation; lorsque l'on fait varier la longueur $L_{\ell}$ de la colonne d'eau entre $15 \mathrm{~cm}$ et $5 \mathrm{~cm}$ (une longueur inférieure entraîne la superposition de réflexions secondaires), on constate que le facteur de transmission global apparent $k$ de l'impulsion entre les capteurs $C_{1}$ et $C_{2}$, défini par le rapport des amplitudes des signaux correspondants, peut s'exprimer sous la forme :

$$
k=\frac{S_{2}}{S_{1}} \sim k_{0}\left(1-b . L_{\ell}\right)
$$

où $k_{0}$ est la valeur de $k$ extrapolée pour une longueur nulle de la colonne d'eau et $b$ le coefficient d'atténuation linéique de l'impulsion dans la colonne d'eau; expérimentalement, $b \sim 0,02 \mathrm{~cm}^{-1}$ et $k_{0} \sim 0,16$.

L'étalement correspondant est présenté sur la figure 3 où l'on a porté le délai de transmission global

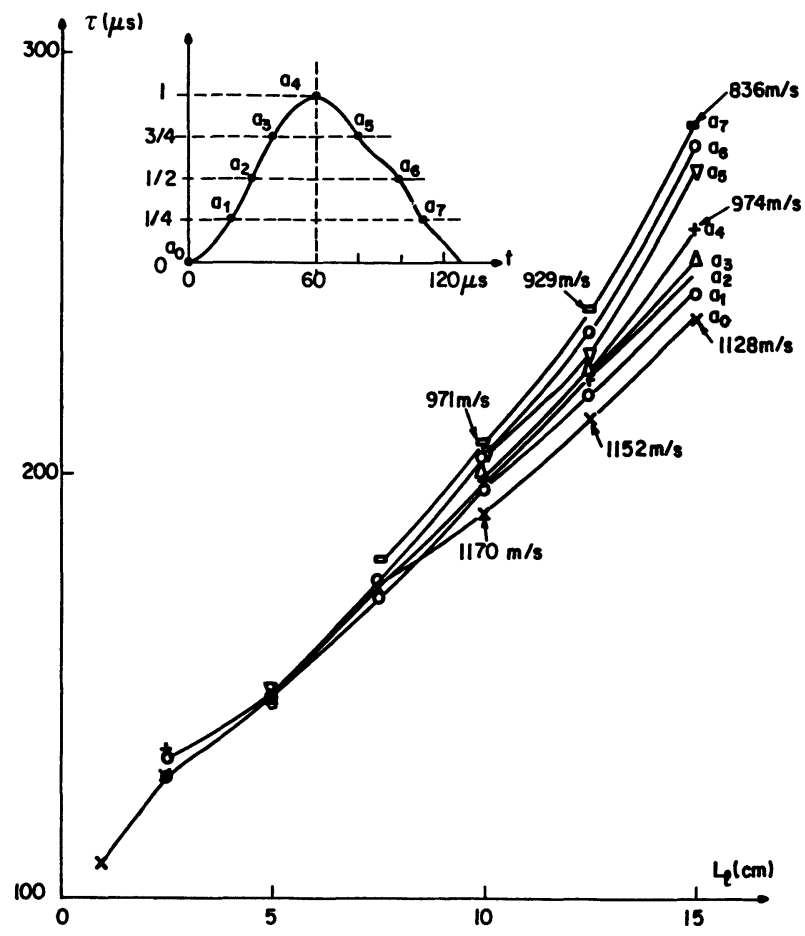

Fig. 3. - Délai de transmission $\tau$ entre les deux capteurs d'impulsions de compression, en fonction de la longueur $L$ de la colonne d'eau, paramétré par le point considéré de l'impulsion.

[Transmission delay $\tau$, between the two transducers, of compression pulses versus the length $L$ of the water column, for different points of the pulse.]

apparent $\tau$ entre les deux capteurs en fonction de $L_{\ell}$, pour différents points de l'impulsion.

La vitesse de transmission $C_{\ell}$ dans la colonne d'eau peut s'exprimer ainsi :

$$
C_{\ell}=\frac{L_{\ell}}{\tau-\left(L_{1}+L_{2}\right) / C_{\mathrm{b}}} .
$$

Considérons, en particulier, la vitesse de transmission du maximum de l'impulsion pour une longueur de colonne d'eau de $10 \mathrm{~cm}$, valeur proche de celles utilisées au cours des différents essais; on obtient, sur la figure 3, $\tau \sim 200 \mu$ s d'où $C_{\ell} \sim 1050 \mathrm{~m} / \mathrm{s}$.

L'expression (4) conjuguée à (5) dans laquelle on prend $30 \mathrm{~mm}$ pour la valeur moyenne de l'épaisseur $h$, conduit à $C_{\ell} \sim 1034 \mathrm{~m} / \mathrm{s}$, valeur proche de la valeur expérimentale précédente.

3.4 Transmission aUX InTERFACES. - D'après la théorie élémentaire sur la propagation dans les milieux élastiques homogènes, une onde de pression plane $p_{\mathrm{i}}$ qui se propage dans un milieu $A$ et atteint sous incidence normale une interface plane avec un milieu $B$, se partage en une onde réfléchie $p_{\mathrm{r}}$ dans le milieu A et une onde transmise $p_{\mathrm{t}}$ dans le milieu $\mathrm{B}$, dans des proportions qui ne dépendent que des valeurs relatives des impédances acoustiques $Z_{\mathrm{A}}=(\rho C)_{\mathrm{A}}$ et 
$Z_{\mathrm{B}}=(\rho C)_{\mathrm{B}}$ de ces deux milieux. Les coefficients de réflexion $K_{\mathrm{r}}$ et de transmission $K_{\mathrm{t}}$ des ondes de pression s'écrivent :

$$
\begin{gathered}
K=\frac{p_{\mathrm{r}}}{p_{\mathrm{i}}}=\frac{Z_{\mathrm{B}}-Z_{\mathrm{A}}}{Z_{\mathrm{B}}+Z_{\mathrm{A}}}=\frac{1-Z_{\mathrm{A}} / Z_{\mathrm{B}}}{1+Z_{\mathrm{A}} / Z_{\mathrm{B}}}, \\
K_{\mathrm{t}}=\frac{p_{\mathrm{t}}}{p_{\mathrm{i}}}=1+K_{\mathrm{r}}=\frac{2 Z_{\mathrm{B}}}{Z_{\mathrm{B}}+Z_{\mathrm{A}}}=\frac{2}{1+Z_{\mathrm{A}} / Z_{\mathrm{B}}} .
\end{gathered}
$$

On remarque que la réflexion se fait sans, ou avec, changement de signe selon que $Z_{B}$ est plus grand, ou plus petit, que $Z_{\mathrm{A}}$, alors que la transmission se fait toujours sans changement de signe; dans le cas particulier d'une surface libre, où $Z_{\mathrm{B}}$ est pratiquement nul, la réflexion est totale avec changement de signe.

Kenner [8] a montré expérimentalement que ces relations s'appliquent bien au cas de la transmission entre une barre solide élastique et un liquide contenu dans un tube mince, pour des impulsions de compression de 30 et $70 \mu$ s de durée. Dans notre cas, la flexibilité et la nature visco-élastique des parois, ainsi que leur épaisseur relativement importante, rendent les phénomènes plus complexes; on peut cependant obtenir un ordre de grandeur des coefficients de transmission à partir des mesures précédentes, en faisant l'hypothèse que les relations de base (10) et (11) s'appliquent encore si l'on remplace l'impédance acoustique vraie $Z_{\ell}$ du liquide par une impédance apparente $Z_{\ell}^{\prime}$ (liée à la compressibilité apparente) proche de et inférieure à $Z_{p}$.

Soient $p_{\mathrm{i} 1}, p_{\mathrm{r} 1}$ et $p_{\mathrm{t} 1}$ les impulsions incidente, réfléchie et transmise à l'interface $\mathrm{I}_{1}$ et $p_{\mathrm{i} 2}, p_{\mathrm{r} 2}$ et $p_{\mathrm{t} 2}$ leurs homologues à l'interface $\mathrm{I}_{2} ;$ si $Z_{\mathrm{b}}$ est l'impédance acoustique des barres, les coefficients de transmission $K_{\mathrm{t} 1}$ en $\mathrm{I}_{1}$ et $K_{\mathrm{t} 2}$ en $\mathrm{I}_{2}$ sont :

$$
\begin{aligned}
K_{\mathrm{t} 1}=\frac{p_{\mathrm{t} 1}}{p_{\mathrm{i} 1}} & =\frac{2 Z_{\ell}^{\prime}}{Z_{\mathrm{b}}+Z_{\ell}^{\prime}}=2 \frac{Z_{\ell}^{\prime} / Z_{\mathrm{b}}}{1+Z_{\ell}^{\prime} / Z_{\mathrm{b}}} \\
K_{\mathrm{t} 2}=\frac{p_{\mathrm{t} 2}}{p_{\mathrm{i} 2}} & =\frac{2 Z_{\mathrm{b}}}{Z_{\mathrm{b}}+Z_{\ell}^{\prime}}= \\
& =2 \cdot \frac{1}{1+Z_{\ell}^{\prime} / Z_{\mathrm{b}}}=2-K_{\mathrm{t} 1} .
\end{aligned}
$$

De plus, la relation (8) nous permet d'écrire :

$$
a_{1} \cdot a_{2} \cdot K_{\mathrm{t} 1} \cdot K_{\mathrm{t} 2}=k_{0} .
$$

Le rapport des impédances acoustiques $Z_{p}$ de l'eau et $Z_{\mathrm{b}}$ d'une barre d'aluminium est :

$Z_{\ell} / Z_{\mathrm{b}}=\frac{(\rho \cdot C)_{\ell}}{(\rho \cdot C)_{\mathrm{b}}}=\left[\left(\rho / \rho_{\mathrm{b}}\right) \cdot\left(B / E_{\mathrm{b}}\right)\right]^{1 / 2} \sim 0,11$

$Z_{\ell}^{\prime} / Z_{\mathrm{b}}$, inférieur à $Z_{\ell} / Z_{\mathrm{b}}$, est lui-même nettement inférieur à 1 . On obtient alors, de (12) et (13), compte tenu des valeurs expérimentales de $a_{1}, a_{2}$ et $k_{0}$ : $K_{\mathrm{t} 1} \sim 0,09, K_{\mathrm{t} 2} \sim 1,9$ et le coefficient de réflexion sur $\mathrm{I}_{2}: K_{\mathrm{r} 2}=\left(K_{\mathrm{t} 2}-1\right) \sim 0,9$.
La figure 4 représente schématiquement la tension qui, en l'absence de cavitation, apparaîtrait de part et d'autre de l'interface $I_{2}$ à des intervalles de temps égaux à partir de l'instant où l'impulsion de tension incidente atteint cette interface; cette tension résulte en chaque point de la composition des valeurs locales instantanées de l'impulsion incidente et de l'impulsion due à sa réflexion sur $\mathrm{I}_{2}$ qui est elle-même une impulsion de tension.

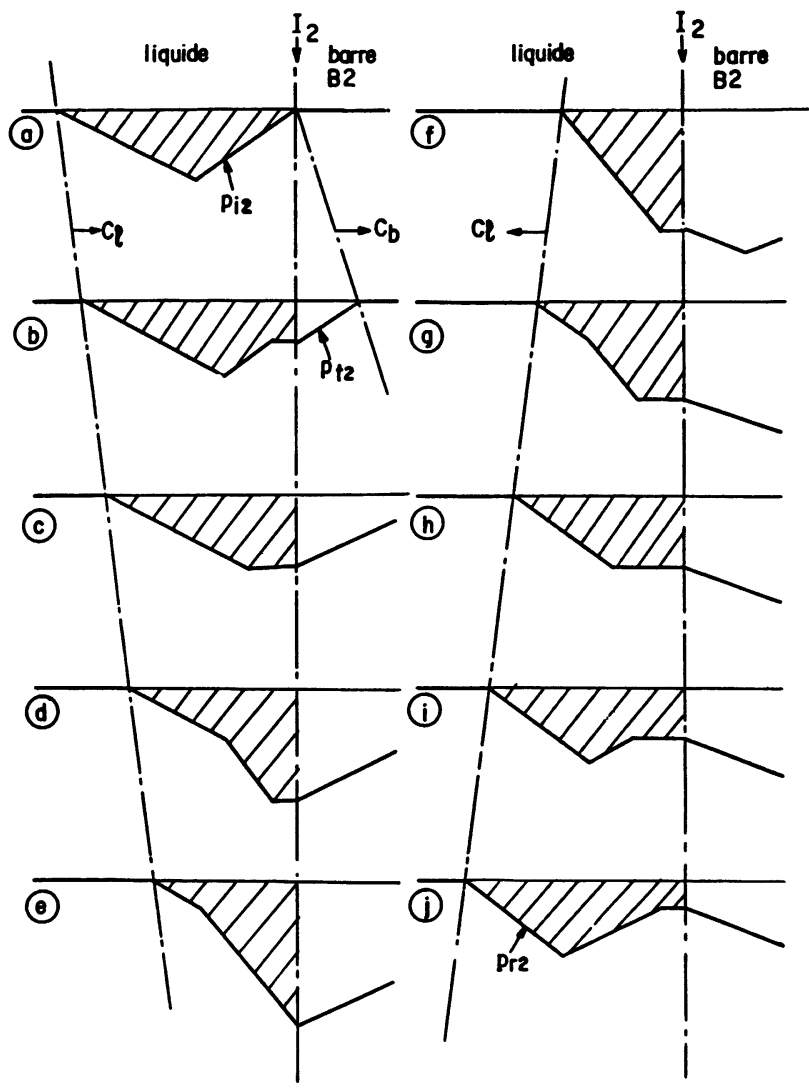

Fig. 4. - Transmission d'une impulsion triangulaire fictive à l'interface entre le liquide et la barre réceptrice, en l'absence de cavitation.

[Transmission of a fictitious triangular pulse at the interface between the liquid and the receiving rod, without cavitation effects.]

On notera, en particulier, que :

- la tension transmise dans la barre réceptrice est à chaque instant égale à la tension $p_{2}$ dans le liquide au niveau de $\mathrm{I}_{\mathbf{2}}$ :

$$
p_{\mathrm{t} 2}=p_{2}=p_{\mathrm{i} 2}+p_{\mathrm{r} 2}=\left(1+K_{\mathrm{r} 2}\right) p_{\mathrm{i} 2} \sim 1,9 p_{\mathrm{i} 2} \text {. }
$$

- la tension dans le liquide prend sa valeur maximum au niveau de $\mathrm{I}_{2}$, à l'instant où (Fig. 4e) la crête de l'impulsion incidente atteint cette interface.

De la même façon, en présence de cavitation, c'est la tension effectivement supportée par le liquide qui constituera la valeur du maximum de l'impulsion de tension transmise dans la barre et sera détectée par le 
capteur $\mathrm{C}_{2}$ (à faible atténuation $a_{2}$ près entre $\mathrm{I}_{2}$ et $\mathrm{C}_{2}$ ).

\section{Résultats expérimentaux.}

4.1 Déroulement D'Un eSSAI. - Tous les essais sont effectués sur de l'eau simplement distillée, préalablement reposée plusieurs heures après emplissage de la chambre d'essais. Ils sont regroupés en séries et, à l'intérieur d'une série, ils sont effectués, avec la même masse d'excitation, sur le même échantillon d'eau. L'intervalle de temps entre deux essais consécutifs d'une même série est généralement de quelques minutes; sa valeur minimum est d'environ $2 \mathrm{~min}$, correspondant au délai nécessaire pour effectuer le contrôle du résultat sur oscilloscope, l'enregistrement graphique et le réarmement de l'acquisition.

Chaque essai se traduit par l'enregistrement graphique des signaux $s_{1}$ et $s_{2}$ des deux capteurs $C_{1}$ et $\mathrm{C}_{2}$. Afin de mettre en évidence les caractéristiques principales d'un enregistrement, on a représenté sur la figure 5a l'enregistrement fictif correspondant à

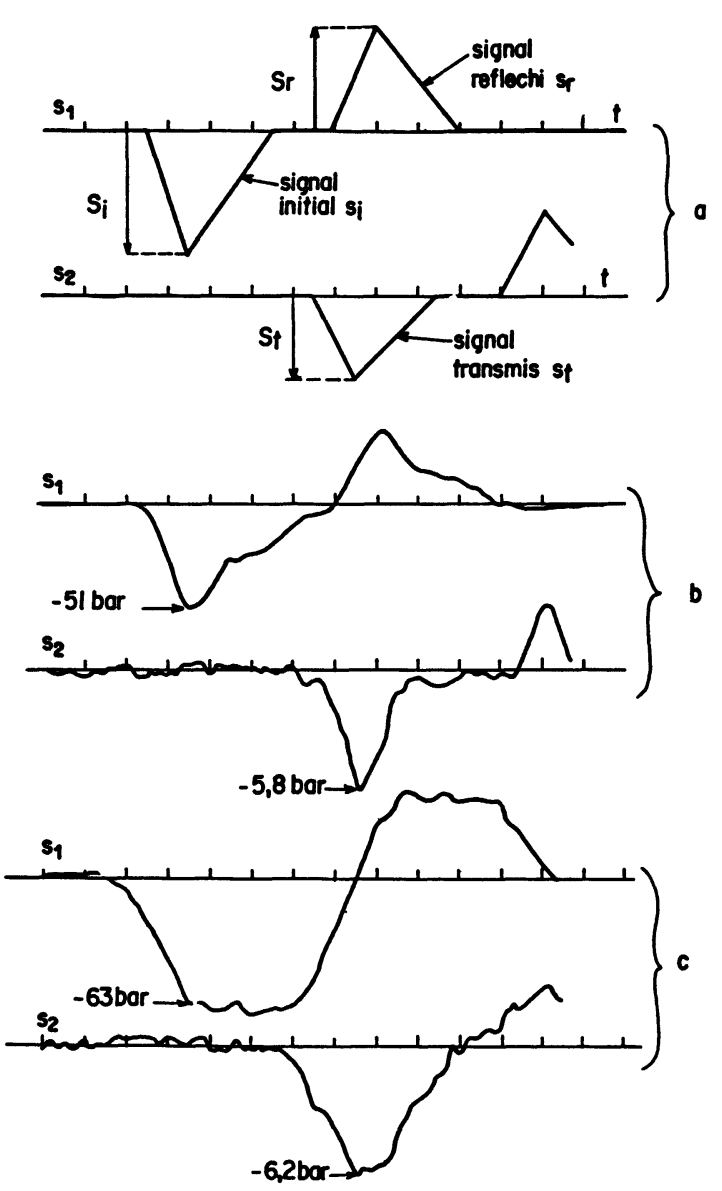

Fig. 5. - Signaux $s_{1}$ et $s_{2}$ des deux capteurs $C_{1}$ et $C_{2}$ : a) signaux fictifs; b) signaux dus à une masselotte de $1 \mathrm{~cm}$ de long; c) signaux dus à une masselotte de $4 \mathrm{~cm}$ (échelle de temps : $30 \mu$ s/division).

[Signals $s_{1}$ and $s_{2}$ of the two transducers $C_{1}$ and $C_{2}$ : a) fictitious signals; b) signals obtained with a weight $1 \mathrm{~cm}$ long; c) signals obtained with a weight $4 \mathrm{~cm}$ long (time scale : $30 \mu \mathrm{s}$ /division).] une impulsion triangulaire de tension transmise sans déformation. Au-dessous, (Figs. 5b et 5c) sont reportés deux enregistrements typiques réels correspondant à deux séries d'essais différentes.

L'enregistrement de la figure $5 \mathrm{~b}$ correspond à l'essai 29 de la figure 7; sa forme est proche de celle de la figure 5a, et reproduit bien les caractéristiques principales de cette dernière. L'enregistrement de la figure $5 \mathrm{c}$ correspond à l'essai 21 de la figure 8 ; la durée plus longue de l'impulsion initiale entraîne un recouvrement partiel de celle-ci par sa réflexion sur $I_{1}$. On remarquera que, même pour des $S_{\mathrm{i}}$ de l'ordre de -50 bar, le bruit est encore appréciable sur $\mathrm{s}_{2}$, tandis qu'on voit apparaître une déformation de $s_{t}$, manifeste par la réduction de sa durée.

4.2 ReLATION ENTRE AMPLITUDE TRANSMISE ET AMPLITUDE INCIDENTE. - Si on porte sur un graphique (Figs. 6, 7, 8) l'amplitude $S$ du signal transmis en fonction de celle $S_{\mathrm{i}}$ du signal incident, on constate que la dispersion des résultats est importante. Les essais sont numérotés suivant l'ordre chronologique. A certains points est associée une représentation des formes des impulsions $s_{i}$ et $s_{t}$ (respectivement en traits pleins et en traits interrompus). L'amplitude relative a été affectée d'un facteur constant pour la série et le délai affecté d'un décalage également constant, de sorte que les impulsions de faible amplitude (amplitude telle que le signal transmis ne soit pas affecté de déformation appréciable due à la cavitation, comme on le verra plus loin) coïncident.

La figure 6 est typique des premières séries d'essais : les essais à faible amplitude initiale $\left(\left|S_{\mathrm{i}}\right|<20\right.$ bar $)$ se groupent autour d'une droite (D) passant par l'origine, qui correspond à un coefficient $k$ constant de l'ordre de 0,14; pour des amplitudes supérieures, les essais se placent au-dessous de cette droite. De plus, bien que l'essai 18, par exemple, corresponde à une amplitude initiale plus faible que l'essai 17, il se situe plus loin de (D); l'essai suivant 19, de même amplitude initiale que l'essai 18 , aboutit au contraire à une réduction de l'écart à $(D)$. Ce phénomène n'apparaît pas dans la succession des essais 20 à 22 . Il semble que l'essai 17 ait provoqué un début de cavitation, dont l'effet sur l'atténuation a diminué entre les essais 18 et 19 ; par contre, la cavitation entraînée par les essais 20 et 21 a entraîné un effet cumulatif sur l'atténuation de l'essai 22 . On peut en conclure que l'intervalle de temps entre ces derniers essais, de l'ordre de $3 \mathrm{~min}$, est insuffisant pour assurer des mesures reproductibles, au moins aux tensions élevées : il devient un paramètre des résultats.

Les séries ultérieures d'essais ont été effectuées en tenant compte de ces premières constatations et en élargissant la gamme des amplitudes. Les figures 7 et 8 sont représentatives de ces séries; les intervalles de temps entre essais consécutifs sont généralement plus longs, et il s'ensuit que les tensions maximales transmises sont nettement plus élevées. Dans les deux 


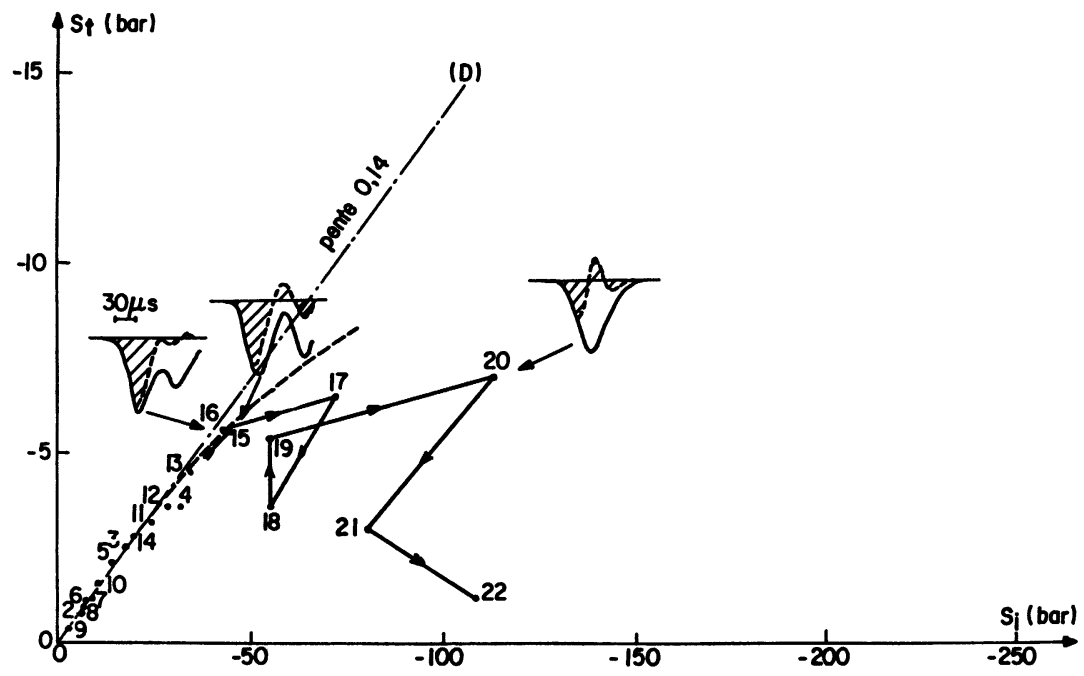

Fig. 6. - Amplitude $S_{\mathrm{t}}$ du signal transmis dans la barre réceptrice en fonction de l'amplitude $S_{\mathrm{i}}$ du signal dans la barre émettrice. Longueur de la colonne d'eau $L=10 \mathrm{~cm}$. Masselotte en laiton de $1 \mathrm{~cm}$ de long.

[Amplitude $S_{\mathrm{t}}$ of the signal transmitted in the receiving rod versus the amplitude $S_{\mathrm{i}}$ of the signal in the emitting rod. Length of the water column $L=10 \mathrm{~cm}$. Brass weight $1 \mathrm{~cm}$ long.]

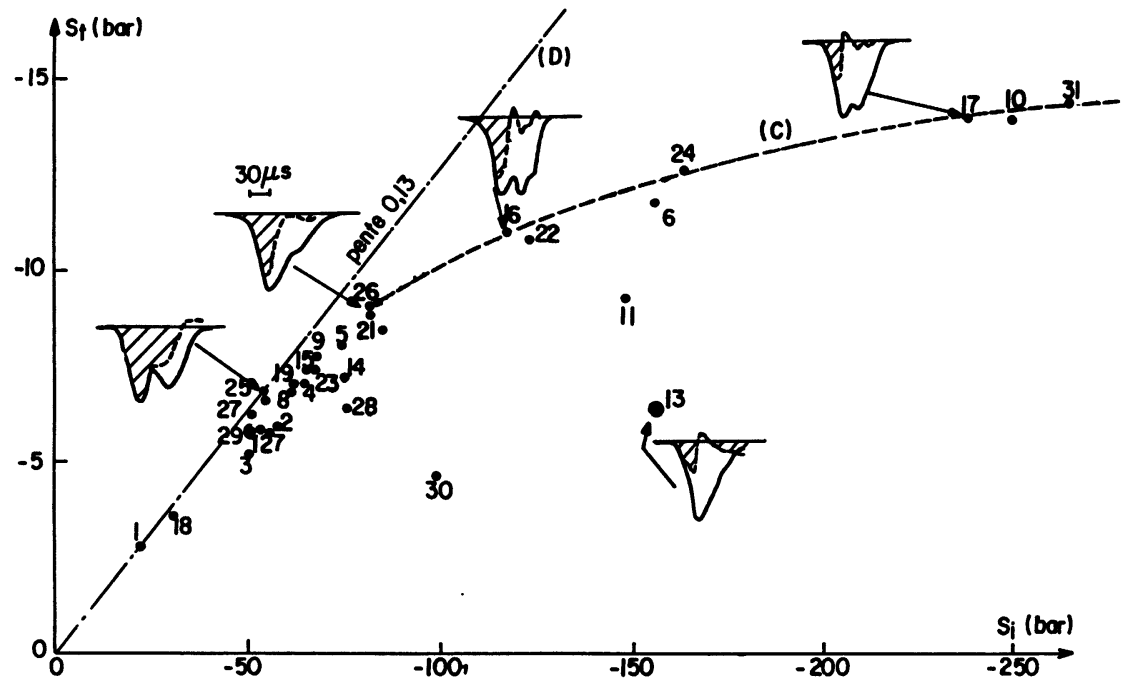

Fig. 7. $-S_{\mathrm{t}}$ en fonction de $S_{\mathrm{i}} . L=13 \mathrm{~cm}$. Masselotte en laiton de $1 \mathrm{~cm}$ de long.

$\left[S_{\mathrm{t}}\right.$ versus $S_{\mathrm{i}} . L=13 \mathrm{~cm}$. Brass weight $1 \mathrm{~cm}$ long.]

séries, la longueur de la colonne d'eau est identique, mais la forme de l'impulsion incidente est différente, la masse excitatrice étant elle-même différente. Quelques points représentatifs sont cerclés : ils correspondent à des essais qui ont été précédés d'un essai à amplitude plus élevée (non numéroté) qui n'a pu être enregistré du fait d'un déclenchement intempestif des enregistreurs; ce fait explique la valeur relativement faible de la tension transmise correspondante, ainsi que lors du ou des essais ultérieurs proches dans le temps.

La figure 7 correspond à des impulsions dont le temps de montée de tension initiale est pratiquement constant et voisin de $25 \mu \mathrm{s}$; la durée et la forme de la descente varient suivant les essais, probablement en fonction de la manière dont s'établit le contact entre la masse et la butée. On remarque tout d'abord que, au-delà de $S_{\mathrm{i}} \sim-50$ bar, les points se détachent de (D), et restent sous une courbe-enveloppe (C) qui correspond à un $k$ décroissant à mesure que les $\left|S_{\mathrm{i}}\right|$ croissent. La tension maximale transmise est de - 14,4 bar pour la tension maximale incidente de - 260 bar, ce qui correspond à un $k$ de 0,055 , alors qu'à faible amplitude, ce coefficient (pente de (D)) est de 0,13 .

La figure 8 correspond à des impulsions dont le temps de montée et la durée sont supérieurs à ceux des impulsions de la figure 7; ce temps de montée 


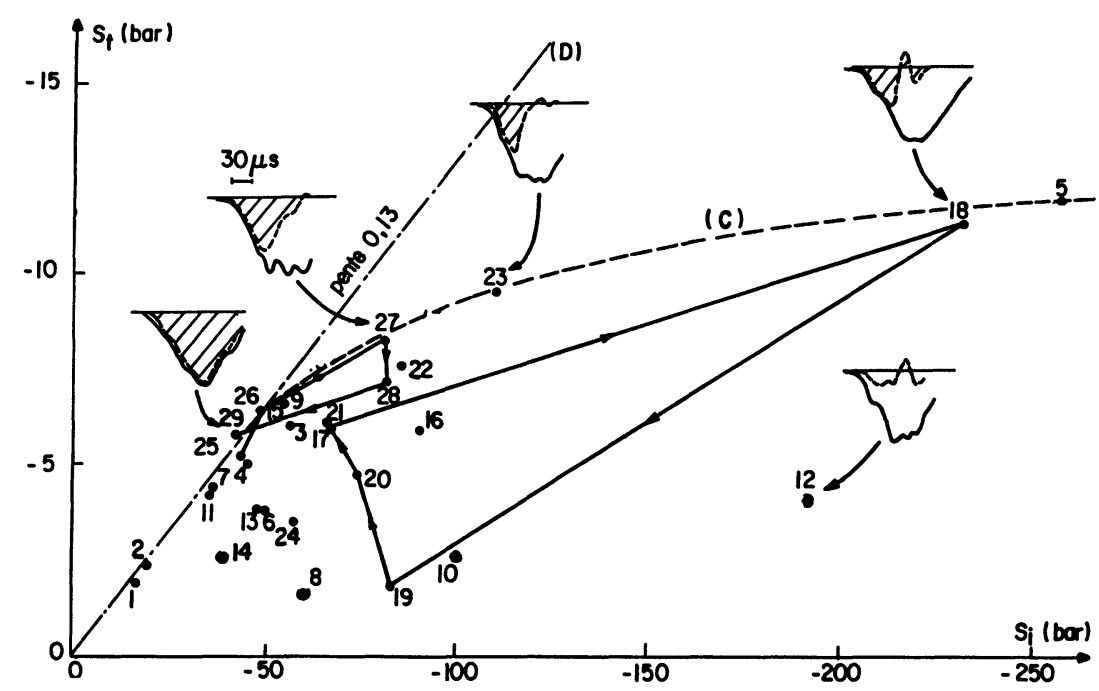

Fig. 8. $-S_{\mathrm{t}}$ en fonction de $S_{\mathrm{i}} . L=13 \mathrm{~cm}$. Masselotte composite.

$\left[S_{\mathrm{t}}\right.$ versus $S_{\mathrm{i}} . L=13 \mathrm{~cm}$. Composite weight.]

est non constant et varie entre 40 et $60 \mu \mathrm{s}$. Du fait de la constitution fractionnée de la masse excitatrice, des déformations apparaissent sur le front de montée des impulsions, et nous serviront par la suite à préciser la nature des déformations subies au cours de la transmission. On remarque que, si la droite (D) a la même pente que sur la figure précédente, l'enveloppe (C) approche plus vite une asymptote, de sorte que le $S_{\mathrm{t}}$ maximal, pour $S_{\mathrm{i}} \sim-250$ bar, n'est plus ici que de - 12 bar et correspond à une valeur de $k$ de 0,048 . On a souligné en traits gras deux boucles, constituées par la succession des points 17 à 21 et 25 à 29, analogues à la boucle des points 17 à 20 de la figure 6 et à celles que l'on peut observer sur la figure 7, dues vraisemblablement à la résorption incomplète de la cavitation.

\subsection{RELATION ENTRE DÉFORMATION DE L'IMPULSION} TRANSMISE ET AMPLITUDE INCIDENTE. - Le rapport entre la durée de l'impulsion transmise et celle de l'impulsion incidente est d'autant plus faible que l'amplitude de cette dernière est plus élevée (cf. encarts des Figs. 6 à 8). Ce phénomène est propre à la transmission d'une impulsion de tension dans la colonne d'eau. En effet, d'une part l'absence complète de transmission lorsqu'on intercale une mince couche d'air entre le liquide et la barre réceptrice élimine l'hypothèse d'une transmission parallèle parasite par l'intermédiaire des joints toriques, des parois de la chambre et des supports du dispositif; d'autre part, les essais comparatifs de transmission d'impulsions de compression ne conduisent jamais à ce phénomène.

La corrélation entre ce rapport et l'amplitude de l'impulsion initiale est mise en évidence par la figure 9, sur laquelle 5 couples $\left(s_{i}, s_{t}\right)$ appartenant aux essais de la figure 7, ont été reproduits, pour des amplitudes $S_{\mathrm{i}}$ de tensions incidentes croissantes. Le couple de gauche correspond à un point voisin de la droite (D) et de l'enveloppe (C) et les autres correspondent à des points voisins de $(C)$ (les $s_{t}$ ont été décalés temporellement (de $-190 \mu \mathrm{s}$ ) relativement aux $\mathrm{s}_{\mathrm{i}}$ de façon à rapprocher les représentations de $s_{i}$ et $s_{t}$ ). On constate que le délai de transmission entre les maxima de $s_{i}$ et $s_{t}$, l'amplitude relative et la durée de l'impulsion transmise sont des fonctions inverses de l'amplitude initiale.

Deux autres caractéristiques de la forme de $s_{t}$ semblent être fonctions directes de l'amplitude maximum de $s_{i}$ : la pente de la décroissance qui suit le maximum et l'amplitude du dépassement éventuel (overshoot) dans le domaine des pressions positives qui suit cette décroissance.

Mentionnons enfin un aspect particulier du couple 24 : le front de montée de $s_{i}$ présente un décrochement net qui se retrouve sur le front de montée de $s_{t}$; le coefficient de transmission de l'amplitude correspondante est de 0,1 , soit nettement supérieur à celui du maximum; de plus, une mesure précise sur l'enregistrement donne un délai de transmission de ce décrochement de $226 \mu \mathrm{s}$, identique au délai observé dans le domaine des $s_{i}$ faibles (points 25 et 26).

Ces constatations suggèrent que la déformation se caractérise principalement par une réduction rapide de l'amplitude du signal après un certain délai compté à partir du début de ce signal, ceci sans modification importante de la fraction du signal antérieure; ce délai est fonction inverse de l'amplitude initiale. Lorsque l'amplitude est suffisamment faible, il est supérieur au temps de montée de l'impulsion et l'amplitude n'est pas affectée (essais pour lesquels les points représentatifs sont à proximité de (D)). Par contre, lorsque l'amplitude dépasse un certain seuil, le délai ci-dessus devient inférieur au temps de montée (points situés sous la courbe $(C)$ ).

La figure 10 confirme le comportement qui vient d'être décrit; elle présente les enregistrements de 


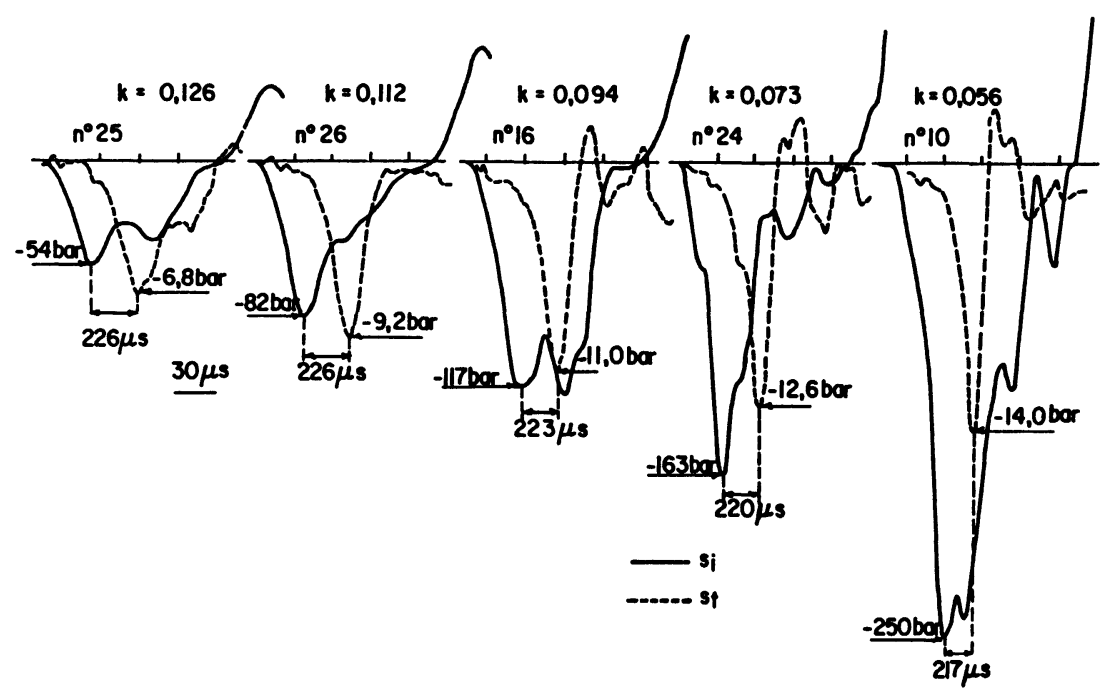

Fig. 9. - Forme de l'impulsion transmise $s_{t}$ en fonction d'amplitudes croissantes de l'impulsion incidente $s_{i}$.

[Shape of the transmitted pulse $s_{t}$ in terms of increasing amplitudes of the incident pulse $s_{i}$ ]

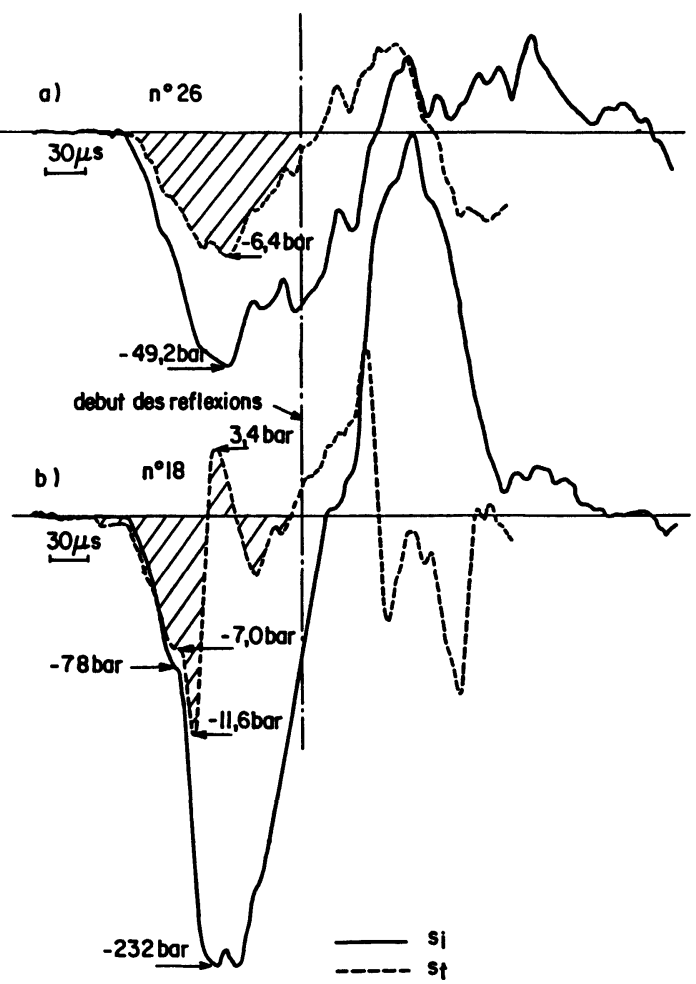

Fig. 10. - Comportement de $s_{\mathrm{t}}$ relativement à $s_{\mathrm{i}}$ à partir de 2 essais de la figure 8 , selon que le point représentatif est : a) voisin de (D); b) voisin de (C), et loin de (D).

[Behaviour of $s_{t}$ in relation to $s_{i}$, for two tests of the figure 8 , depending on whether the corresponding point is : a) near to (D), or b) near to (C) and far from (D).]

deux essais de la figure 8 dont les amplitudes incidentes sont très différentes, le point représentatif $d u$ premier essai étant proche de (D) alors que le point représentatif de l'autre essai est proche de (C) et loin de (D); les fronts de montée des impulsions incidentes des deux essais comportent un palier caractéristique (comme sur la figure 9, les signaux ont été décalés pour faire coïncider les maxima). L'impulsion transmise dans l'essai à forte amplitude a subi une réduction brutale au-delà d'un certain point de son front de montée; par contre, la zone du palier antérieure à cette réduction, mais proche de celle-ci, n'est pas affectée sensiblement.

\section{Discussion.}

La rapidité de l'atténuation qui affecte le front de montée de l'impulsion transmise, lorsque l'amplitude dépasse une certaine valeur, suggère un changement brutal des propriétés du fluide dû à la croissance explosive des microbulles stabilisées en son sein. La persistance de microbulles a été mise en évidence expérimentalement par différentes méthodes : optique [9], acoustique [10] et électrique [11]. Cette croissance est dite vaporeuse, par opposition à la croissance gazeuse qui intervient dans les méthodes statiques et ultrasoniques, car la durée d'application de la tension est bien trop courte pour que des échanges gazeux appréciables aient lieu entre le milieu liquide et l'intérieur de la bulle. L'analyse du problème de l'évolution du rayon d'une bulle sphérique isolée au sein d'un liquide et en équilibre avec lui (par exemple à partir de l'équation de Rayleigh), lorsqu'on applique à ce liquide une tension à pente de montée constante $m$, qui représente assez bien le front de montée de nos impulsions (à l'exception du voisinage de l'origine et du maximum), fait apparaître deux comportements successifs très différents selon que la tension instantanée est inférieure ou supérieure à la tension dite de Blake, fonction inverse du rayon initial : tant que la tension instantanée est inférieure à la tension de Blake, la vitesse de croissance est faible, alors qu'au- 
delà, la loi de croissance du rayon se rapproche d'une loi en $\left(m t^{3}\right)^{1 / 2}$ (solution asymptotique) indépendante du rayon initial. Lorsque $\left(B \alpha K_{\mathrm{g}}\right)$ dépasse l'unité, il en découle une diminution rapide de l'impédance acoustique du milieu et, en conséquence, de l'amplitude du signal transmis.

Soit une impulsion de tension appliquée d'amplitude $P$, de temps de montée $T$ et de pente de montée constante $m=P / T$. Pour établir une relation explicite entre l'amplitude de la tension transmise et ces paramètres, il faut faire une hypothèse sur la densité de distribution des rayons initiaux des microbulles; la plus simple est de supposer que les rayons ont tous la même valeur; on en déduit facilement que l'amplitude transmise est égale à $P$ tant que cette valeur est inférieure au seuil de Blake de ces bulles, et tend, pour un taux de vide initial donné, vers une valeur asymptotique proportionnelle à $(P / T)^{2 / 3}$, donc à $P^{2 / 3}$ pour des impulsions de temps de montée constant. Une hypothèse plus réaliste consiste à supposer une loi de distribution des rayons initiaux $R_{0}$ en $R_{0}^{-\beta}$, où $\beta$ est de l'ordre de 3 à 4, comme l'ont montré expérimentalement Gavrilov [12] pour l'eau ordinaire ou distillée et Medwin [13] pour l'eau de mer; ce dernier explique une telle distribution en arguant qu'elle doit être liée à celle des particules de rayon $r$ en suspension dans le liquide, et donc, en particulier, des aérosols continentaux dont la distribution est en $r^{-3}$ ou $r^{-3,5}$. Dans cette hypothèse, à chaque instant de la croissance de la tension appliquée, seuls les noyaux pour lesquels la valeur instantanée de cette tension est supérieure à la tension de Blake subissent une croissance appréciable; la loi de distribution réelle des rayons des noyaux dont la tension de Blake est supérieure à la tension maximum transmise n'a donc pas besoin d'être connue. On déduit ainsi que cette tension maximum tend, pour un taux de vide initial donné, vers une valeur asymptotique proportionnelle à $P^{0,4}$ à $T$ constant pour $\beta$ de l'ordre de 3,5 .

Si l'on porte les points expérimentaux de la figure 9 sur un graphe logarithmique, on obtient une loi en puissance $2 / 3$ entre les points 25 et 16 , l'exposant diminuant jusqu'à environ $1 / 3$ pour les points situés plus à droite. Un traitement analogue effectué sur les points de la figure 81-A de la thèse de Brown [3], dans laquelle la tension est obtenue par réflexion sur la surface libre de la colonne d'eau, conduit à un exposant compris entre 0,55 et 0,7 environ; les impulsions ont la forme d'une arche de sinusoïde, de durée $40 \mu$ s et l'amplitude varie entre 3,5 et 70 bar. Par contre, les essais de Couzens et Trevena [14], analogues à ceux de Brown, et où les impulsions ont un temps de montée de $50 \mu$ s environ et une amplitude variant entre 4 et 100 bar, conduisent à une courbe (C) qui devient pratiquement horizontale au-delà de 60 bar; cependant, comme il est indiqué page 151 du livre de Temperley et Trevena [15], les impulsions sont provoquées par des balles et des pistons de dimensions variées; il est donc possible que l'effet de l'augmentation de l'amplitude soit compensé par l'effet inverse dû à la diminution de la pente du front de montée.

Pour tous les résultats expérimentaux, une diminution apparente de la valeur de l'exposant aux tensions élevées peut provenir de deux autres effets :

- la durée de l'impulsion transmise devient assez courte pour que la limitation de la bande passante de la chaîne de mesure provoque une atténuation sensible,

- l'amplitude de l'impulsion appliquée devient assez forte pour que la pente plus faible au voisinage de l'origine intervienne appréciablement sur la croissance des bulles.

Ces résultats peuvent aussi s'appliquer aux impulsions de tension dues à la réflexion, à la surface, des ondes de choc engendrées par des explosions sousmarines, dans la mesure où, comme le soulignent Wentzell et Lastman [16], leur temps de montée est fini (mais dépend du type et de la taille de l'explosion, ainsi que de la distance de la source).

\section{Conclusion.}

L'application directe d'une impulsion de tension à une extrémité d'une colonne d'eau nous a permis de mettre en évidence la relation entre tension transmise par cette colonne à son autre extrémité et tension appliquée. Les résultats se comparent qualitativement bien à ceux de Brown [3] qui réalise indirectement la mise en tension par réflexion d'une impulsion de compression à l'extrémité libre d'une colonne d'eau.

L'analyse de la déformation caractéristique de l'impulsion transmise nous a montré que la diminution $\mathrm{du}$ rapport de l'amplitude transmise à l'amplitude appliquée, pour les fortes valeurs de celle-ci, est due à une réduction rapide du signal transmis après un certain délai; la réduction apparaît d'autant plus tôt que l'amplitude appliquée est plus forte.

Ce comportement suggère la croissance explosive des microbulles stabilisées au sein du liquide; un modèle simple basé sur l'équation de Rayleigh est proposé.

\section{Bibliographie}

[1] BRIGGS, L. J., "Limiting negative pressure of water ", J. Appl. Phys. 21 (1950) 721-722.

[2] SiRotyuK, M. G., « Experimental investigation of the growth of ultrasonic cavitation at $500 \mathrm{kc}$ », Sov. Phys. Acoust. 8 (1962) 165-169.
[3] BRown, S. J., "The behavior of tap water under dynamic tensile stressing in a non-flow system ", M. S. Thesis, Pennsylvania University (1967).

[4] Davies, R. M., "A critical study of the Hopkinson pressure bar ", Trans. R. Soc. A 240 (1948) 375-457. 
[5] Paynter, H. M., Fluids Transients in engineering systems, V. L. Streeter, ed. (McGraw Hill) 1961.

[6] Rubinov, S. L., Keller, J. B., « Wave propagation in a visco-elastic tube containing a viscous fluid ", J. Fluid Mech. 88 (1978) 181-203.

[7] Barez, F., Goldsmith, W., Sackman, J. L., « Longitudinal waves in liquid-filled tubes-II $»$, Int. $J$. Mech. Sci. 21 (1979) 223-236.

[8] KenNeR, V. H., "One-dimensional transmission and reflection at solid fluid interfaces ", Proceedings of the Sixth Canadian Congress of Applied Mechanics, Vancouver (Canada) 1977, pp. 239-240.

[9] KelleR, A., "The influence of the cavitation nucleus spectrum on cavitation inception, investigated with a scattered light counting method ", J. Basic Eng. (1972) 917-925.

[10] Medwin, H., "Acoustical determination of bubblesize spectra ", J. Acoust. Soc. Am. 62 (1977) 10411044.
[11] Pyun, J. J., Hammitt, F. G., Keller, A., « Microbubble spectra and superheat in water and sodium, including effects of fast neutron irradiation ", J. Fluid Eng. (1976), pp. 87-97.

[12] Gavrilov, L. R., Free gas content of a liquid and acoustical techniques for its measurement, Sov. Phys. Acoust. 15 (1970) 285-295.

[13] MEDwIN, H., «In situ acoustic measurements of bubble populations in coastal ocean waters $", 75, J$. GeoPhys. Res. 75 (1970) 599-611.

[14] Couzens, D. C. F., Trevena, D. H., « Critical tension in a liquid under dynamic conditions of stressing ", Nature 222 (1969) 473-474.

[15] Temperley, H. N. V., Trevena, D. H., Liquids and their properties (Ellis Norwood) 1978.

[16] Wentzell, R. A., Lastman, G. J., « A study of some experimental results in cavitation ", Phys. Fluids 26 (1983) 638-640. 